\title{
US High-Luminosity LHC Accelerator Upgrade Program (AUP)
}

V. Marinozzi, M. Baldini on behalf of the AUP collaboration Fermilab, APS-TD Magnet Systems Department

FERMILAB-POSTER-20-013-TD

\section{High-Luminosity LHC}

HL-LHC project aims at increasing luminosity from $300 \mathrm{fb}^{-1}$ to $3000-4000 \mathrm{fb}^{-1}$. The project is led by CERN with the support of an international collaboration of 29 institutions in 13 countries, including the United States.

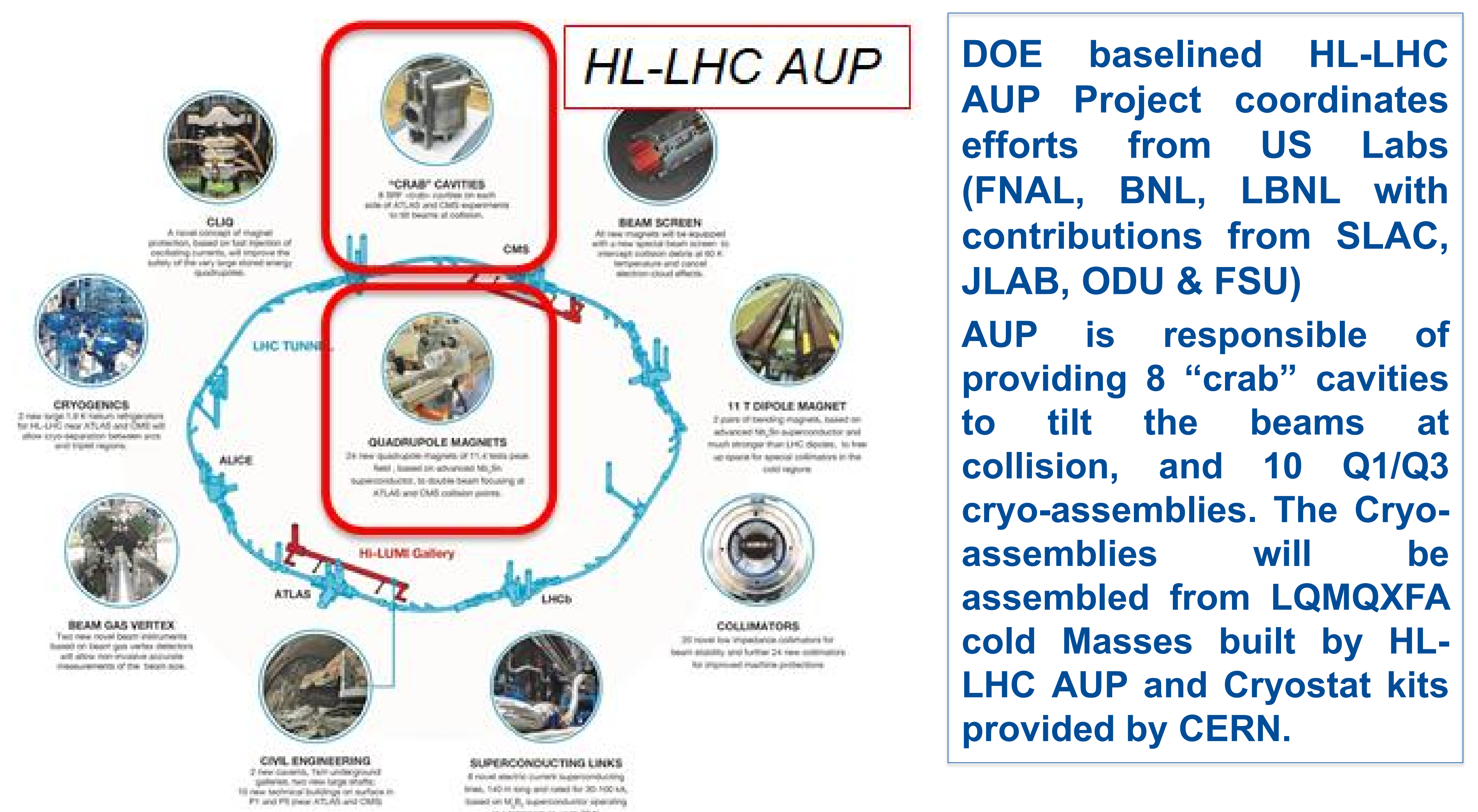

Q1 and Q3 cryo-assembly

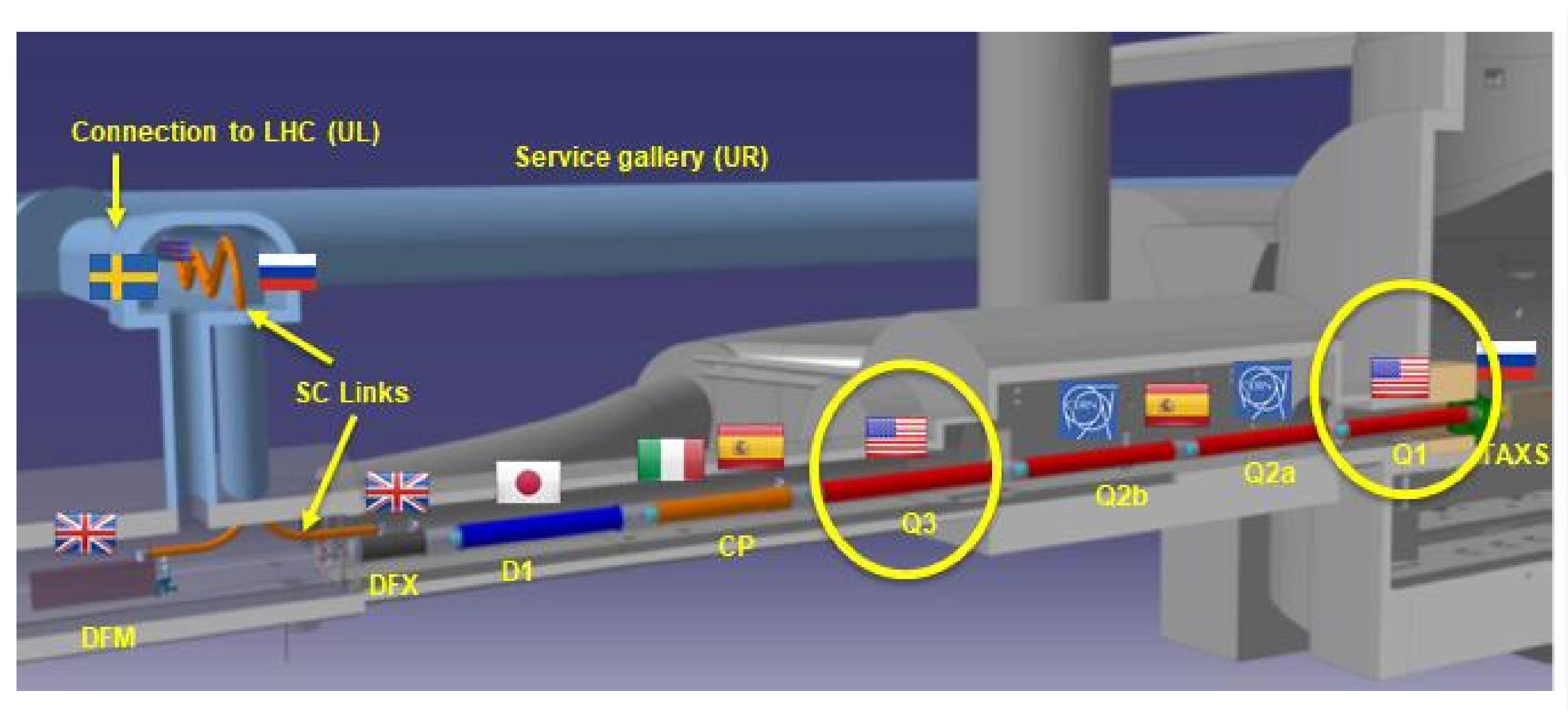

The low-b triplets are made of three optical elements: Q1, Q2, and Q3. Q1/Q3 cold masses contain two $4.2 \mathrm{~m}$ quadrupole magnet responsible of focusing the beams in HL-LHC collisions points (ATLAS and CMS).

HL-LHC Inner Triplet. Q1 and Q3 magnets will be delivered by US AUP project

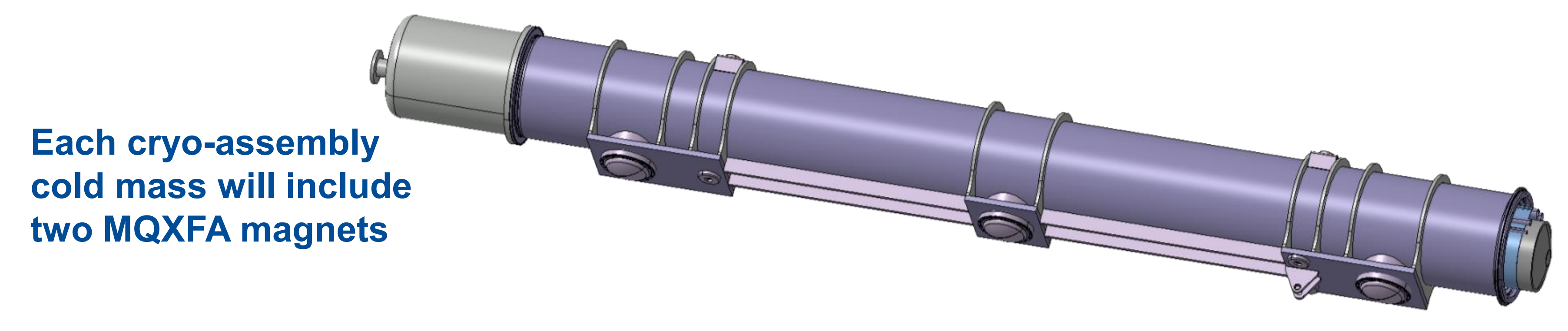

Schedule

The AUP project covers procurement of conductor and parts, cable and coil fabrication, magnet assembly and vertical test, cryomodule assembly and horizontal test. The 10 cryoassemblies (20 magnets) will be delivered by the end of FY2025

HL-LHC AUP Q1/Q3 Cryo-Assemblies Schedule Chart

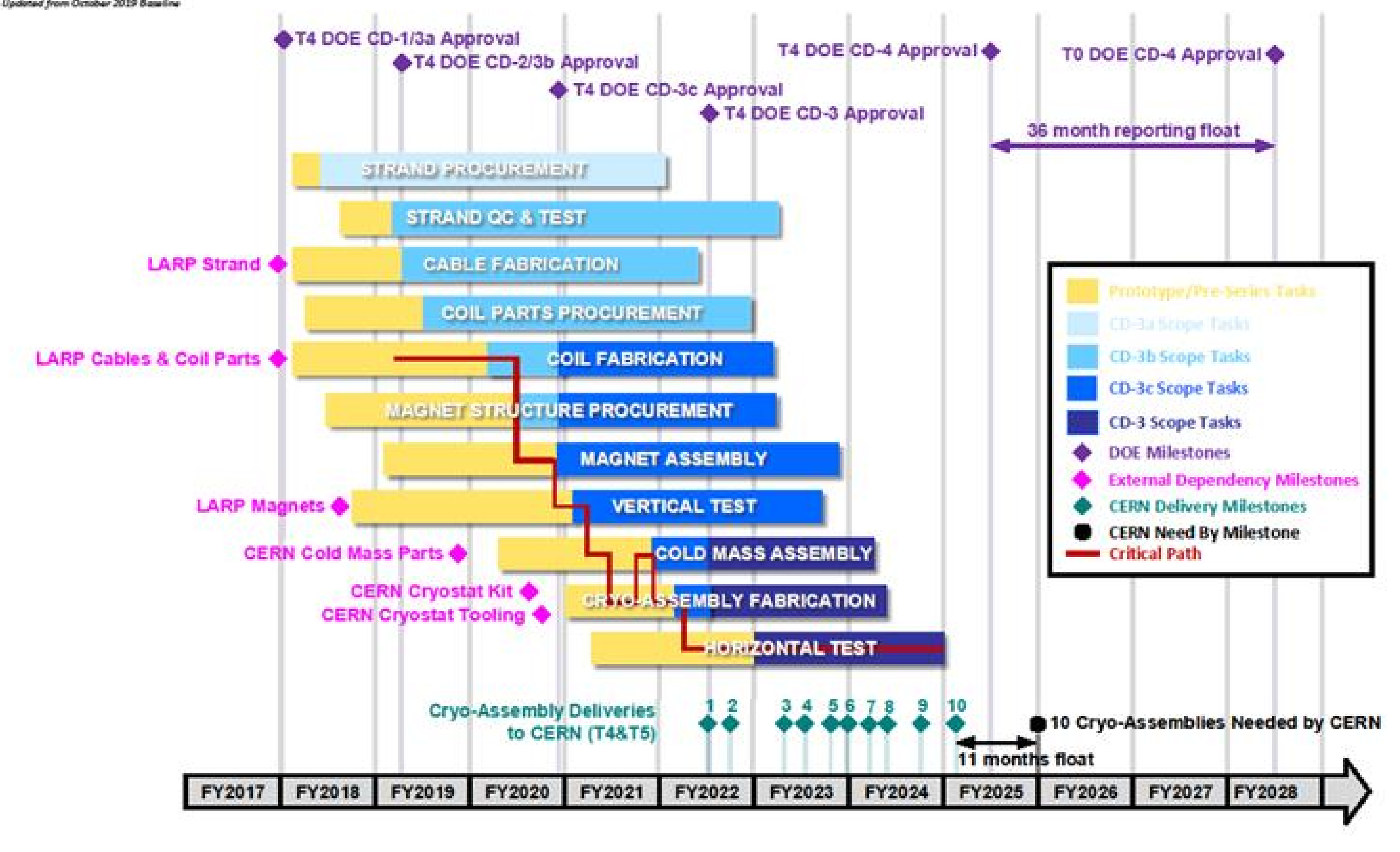

Schedule of the AUP cryo-assemblies delivery

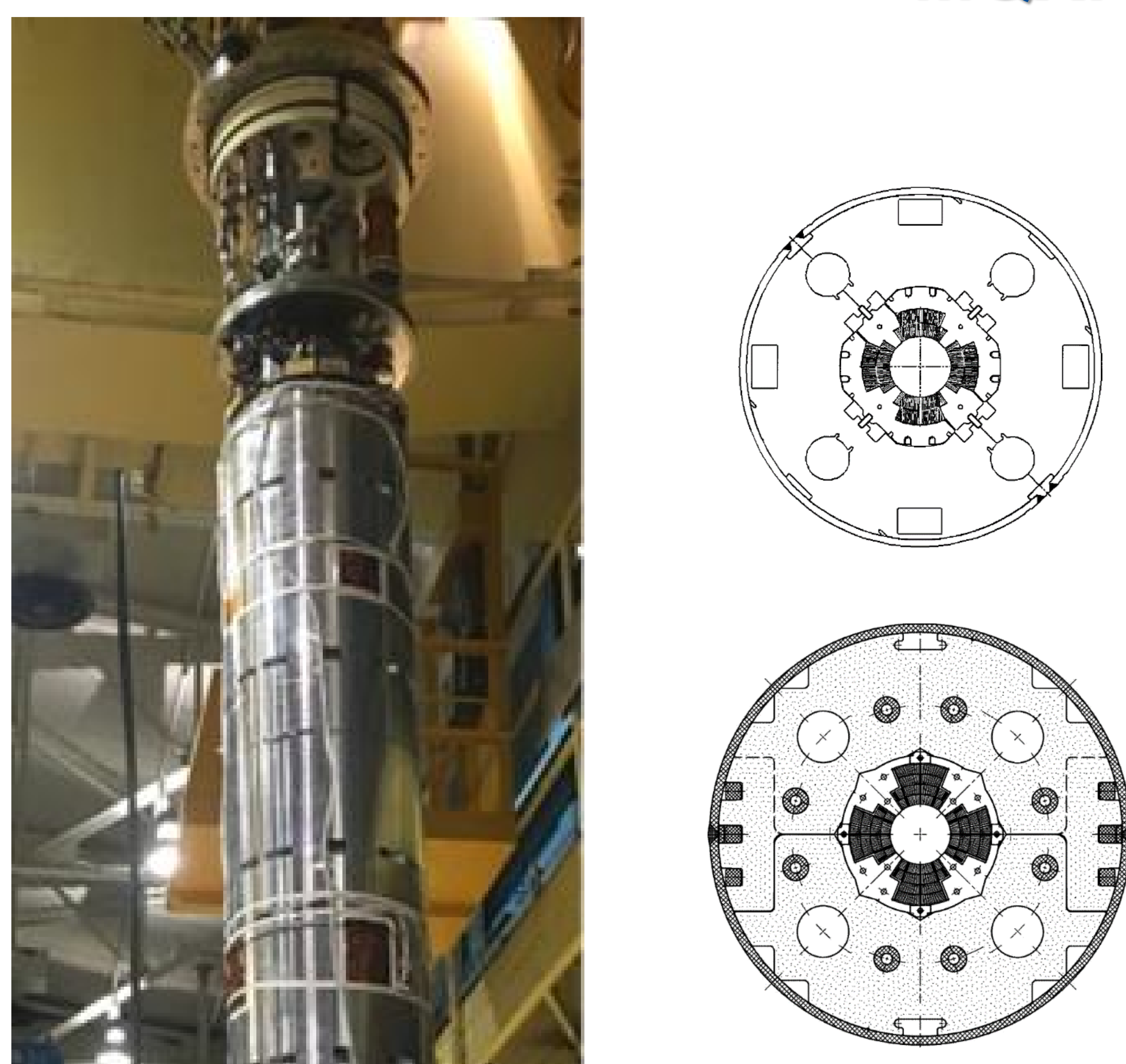

MQXFA magnets

MQXFA magnets are $4.2 \mathrm{~m}$ long with a $150 \mathrm{~mm}$ aperture, and produce a $132.6 \mathrm{~T} / \mathrm{m}$ gradient with $11.4 \mathrm{~T}$ peak field on coils.

MQXFA magnets will be first $\mathrm{Nb}_{3} \mathrm{Sn}$ superconducting magnets installed in an accelerator (together with CERN $11 \mathrm{~T}$ dipole magnets). The AUP project and the LARP program before it, have been leading the R\&D effort to develop those state of the arts accelerator magnets.

Cross section of present LHC triplet quadrupoles
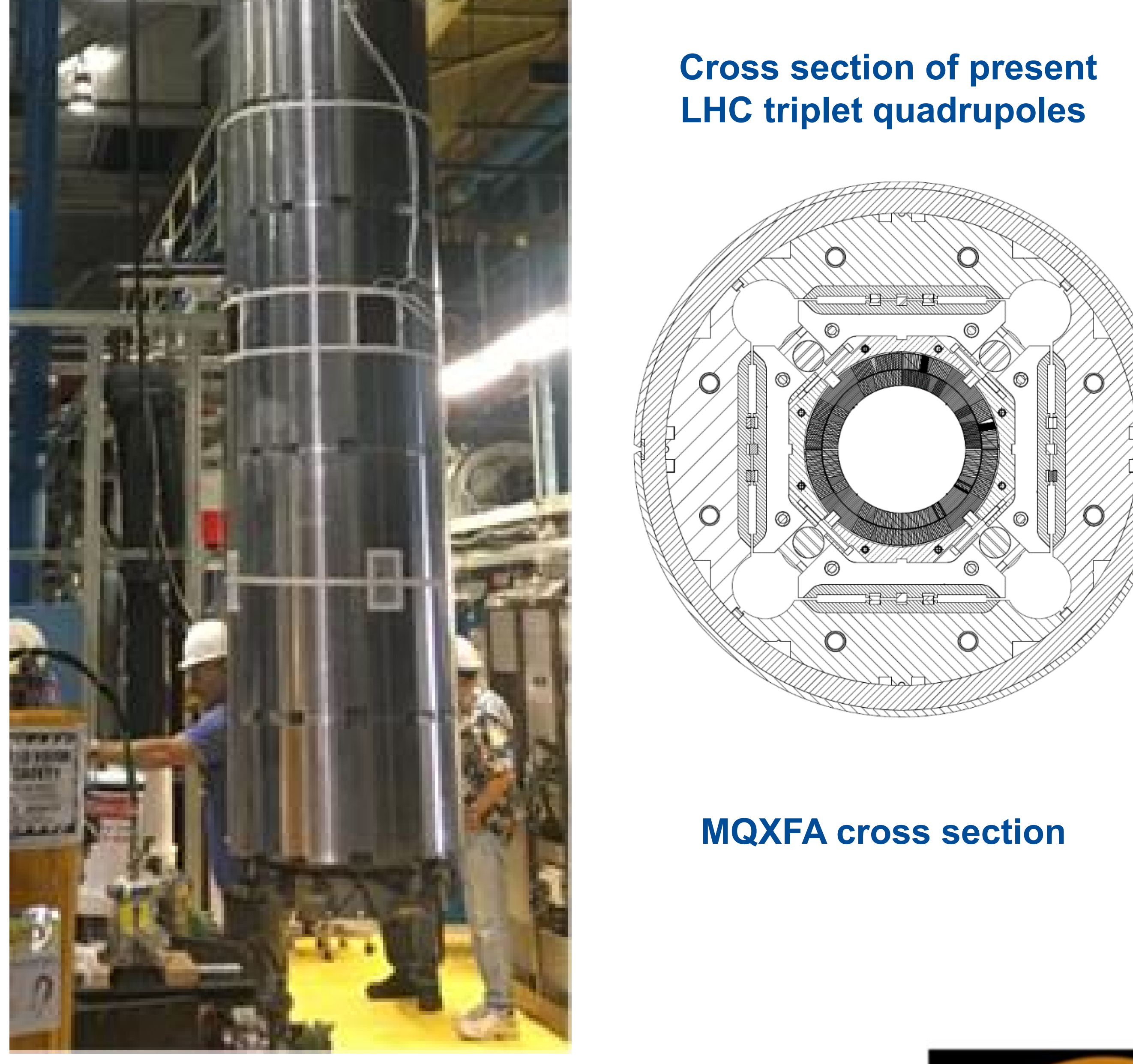

MQXFA cross section

The brittleness of the conductor and the large electromagnetic forces (4 to 6 times higher than the present LHC triplet quadrupoles) are some of the technical challenges present in the fabrication of $\mathrm{Nn}_{3} \mathrm{Sn}$ coil and assembly of MQXFA magnets.

MQXFA magnets are the result of $\sim 20$ years development and represent the maturity of $\mathrm{Nb}_{3} \mathrm{Sn}$ accelerator technology.

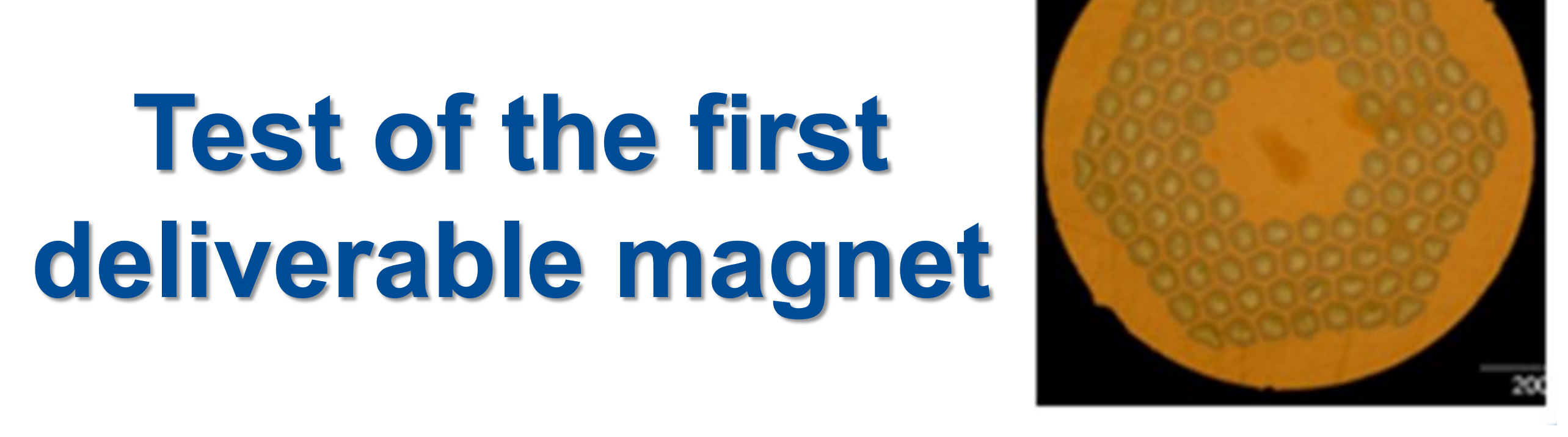

The first MQXFA series magnet has successfully met all the requirements during vertical test @BNL. Prior to that several tests on 4 short models MQXFS and 2 full length MQXFA prototypes have been performed.

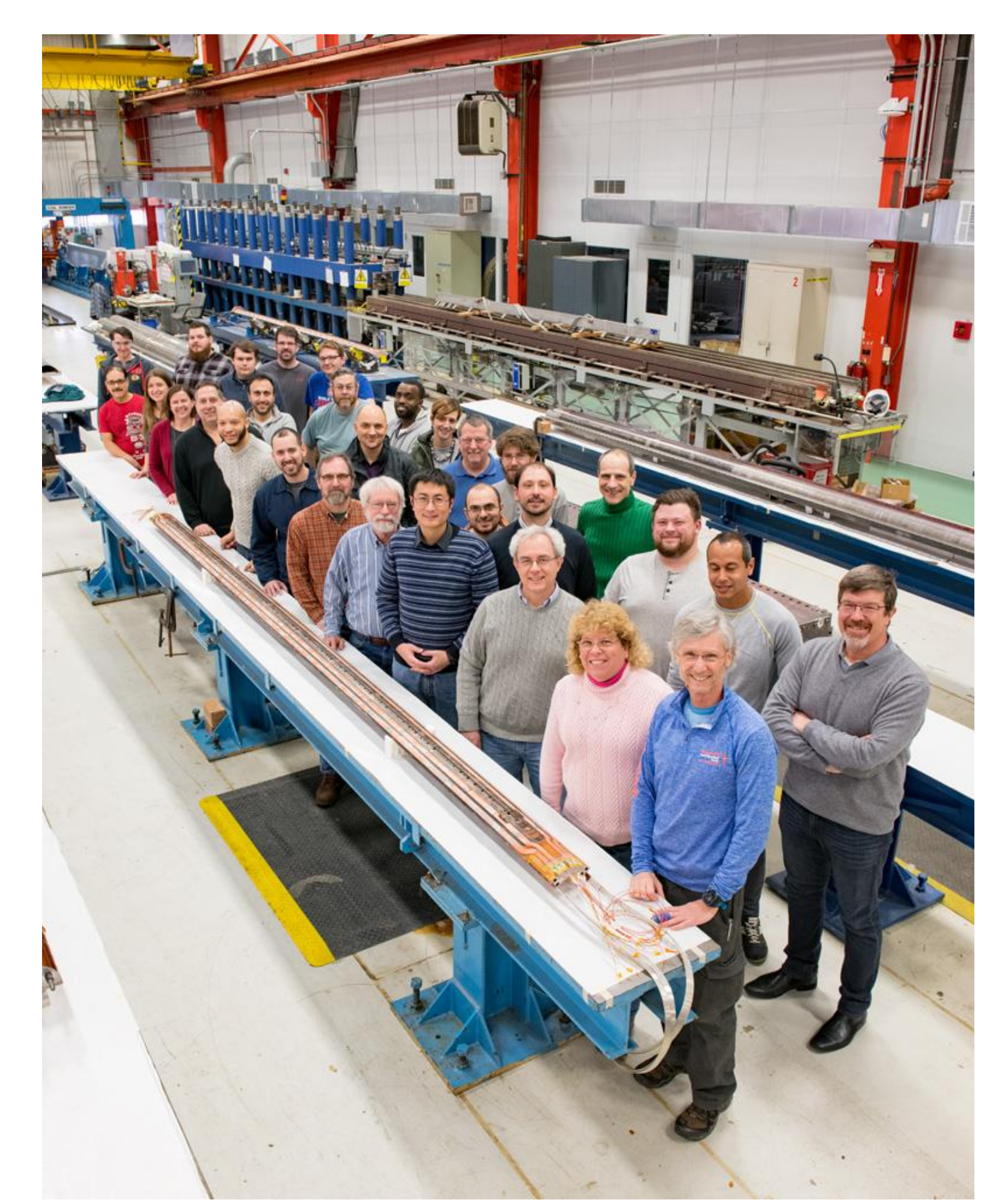

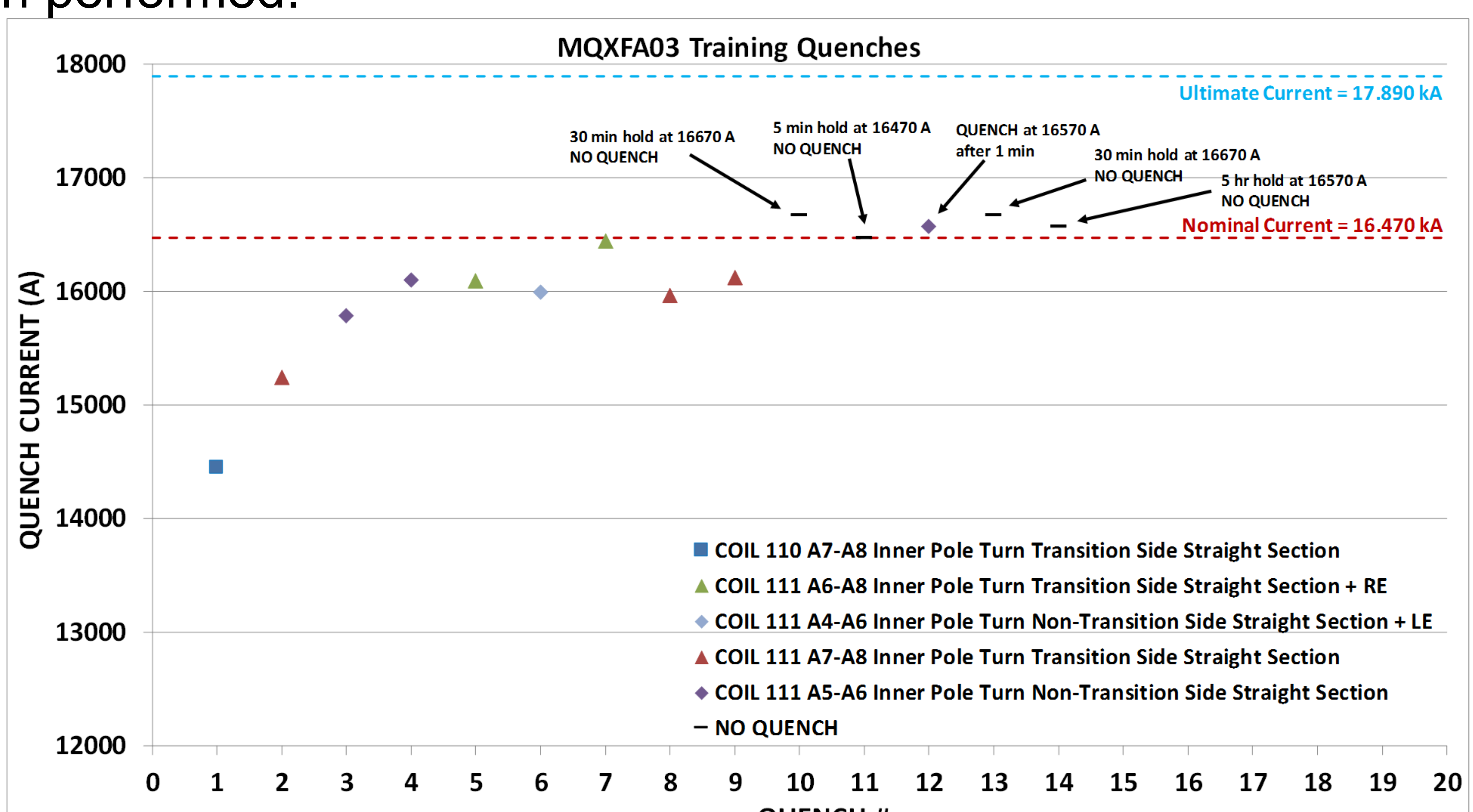

